\title{
ON THE FIRST OCCURRENCE OF VALUES OF A CHARACTER
}

BY

\author{
G. KOLESNIK AND E. G. STRAUS ${ }^{1}$
}

\begin{abstract}
Let $\chi$ be a character of order $k(\bmod n)$, and let $g_{m}(\chi)$ be the smallest positive integer at which $\chi$ attains its $(m+1)$ st nonzero value. We consider fixed $k$ and large $n$ and combine elementary group-theoretic considerations with the known results on character sums and sets of integers without large prime factors to obtain estimates for $g_{m}(\chi)$.
\end{abstract}

1. Introduction. Let $\chi$ be a character of order $k(\bmod n)$, and let $g_{m}(\chi)=$ $n^{1 / \gamma_{m}(x)}$ be the least positive integer at which $\chi$ attains its $(m+1)$ st nonzero value.

Even though we shall not need most of the information, it may be useful to give a brief review of some elementary facts about characters which can be found in most textbooks on the subject. The character group $(\bmod n)$ is isomorphic to the multiplicative group $G(n)$ of reduced residue classes $(\bmod n)$. If we write $n=2^{\alpha_{1}} p_{1}^{\alpha_{1}} \cdots p_{s}^{\alpha_{s}} ; \alpha \geqslant 0, \alpha_{j}>0(j=1, \ldots, s)$ where $p_{j}$ are odd primes, then, by the Chinese remainder theorem, $G(n)$ is the direct product of the groups $G\left(2^{\alpha}\right), G\left(p_{j}^{\alpha_{j}}\right)(j=1, \ldots, s)$. Now $G\left(p_{j}^{\alpha_{j}}\right)$ is cyclic of order $\varphi\left(p_{j}^{\alpha_{j}}\right)=p_{j}^{\alpha_{j}-1}\left(p_{j}-1\right)$ and $G\left(2^{\alpha}\right)=\{1\}$ if $\alpha=0,1$, while if $\alpha>2$, then $G\left(2^{\alpha}\right)$ is the direct product of a group of order 2 and a cyclic group of order $2^{\alpha-2}$. Thus there exists a character of order $k=2^{\beta^{\beta}} q_{1}^{\beta_{1}} \cdots q_{i}^{\beta_{1}}$, where the $q_{j}$ are odd primes, if and only if the following conditions are satisfied:

For every $j=1, \ldots, t$ we have either

$$
p_{i} \equiv 1\left(\bmod q_{j}^{\beta_{j}}\right) \text { for some } i,
$$

or

$$
p_{i}=q_{j} \text { and } \alpha_{i}>\beta_{j} \text { for some } i \text {, }
$$

and either

$$
p_{i} \equiv 1\left(\bmod 2^{\beta}\right) \text { for some } i \text {, }
$$

Received by the editors August 16, 1976.

AMS (MOS) subject classifications (1970). Primary 10H35.

${ }^{1}$ Research of the first author was supported in part by a grant from ACEP, Program for Soviet Emigre Scholars. Research of the second author was supported in part by NSF Grant MPS 71-2884. 
or

$$
\beta=0, \quad \text { or } \beta=1 \text { and } \alpha>1, \quad \text { or } \beta>1 \text { and } \alpha>\beta+1 \text {. }
$$

The group of nonzero values of a character of order $k$ is the group of $k$ th roots of unity. Also the character $\chi(\bmod n)$ of order $k=2{ }^{\beta_{1}} q_{1}^{\beta_{1}} \cdots q_{t}^{\beta_{t}}$ can be expressed uniquely as a product of characters $\chi_{0}, \chi_{1}, \ldots, \chi_{1}(\bmod n)$ of orders $2^{\beta}, q_{1}^{\beta_{1}}, \ldots, q_{t}^{\beta_{t}}$, respectively.

Throughout this paper the modulus $n$ of the characters is assumed fixed and cube free (although some of the results could be extended to more general $n)$. So we shall omit the notation $(\bmod n)$. The symbol $\varepsilon$ stands for a positive number which can be chosen arbitrarily small, although different uses of $\varepsilon$ in the same statement may refer to unequal choices. Since $g_{0}(\chi)=1$ $\left(\gamma_{0}(\chi)=\infty\right)$ holds for all $\chi$ we use the symbol $g_{m}(\chi)$ in the following with the understanding that $m>0$.

In $\$ 3$ we use simple group theoretic facts to obtain estimates of $g_{m}(\chi)$ in terms of various values $g_{l}\left(\chi^{\prime}\right)$ with $l<m$ or ord $\chi^{\prime}<k$. In $\S 4$, we use estimates for character sums and estimates on the number of integers without large prime divisors to get bounds for $g_{1}$ and apply these results to the result of $\$ 3$ to obtain bounds for $g_{m}$ which are useful for values of $m$ which are small compared to $k$. Finally in $\$ 5$ we illustrate a method of estimating the number of integers with few large prime factors to improve the estimates for $g_{m}$ by carrying out some of the details in estimating $g_{2}$.

2. A group theoretic lemma. Let $G$ be a group and $S$ a subset of $G$. Define

$$
S_{l}=\left\{s_{1} s_{2} \cdots s_{l} \mid s_{i} \in S\right\}, \quad l=1,2, \ldots
$$

If $1 \in S$ and $S_{0}=\{1\}$ then $S_{0} \subset S_{1} \subset S_{2} \subset \ldots$

2.1. TheOREM. If $1 \in S$ and $\left|S_{m}\right| \leqslant m$, then $S_{m}$ is a subgroup of $G$ and $S_{m-1}=S_{m}=S_{m+1}=\cdots$.

Proof. The theorem is obvious when $m=1$. Now assume the theorem true for $m-1$. If $S_{m-1} \subsetneq S_{m}$, then $\left|S_{m-1}\right| \leqslant m-1$ and therefore $S_{m-1}$ is a subgroup. Hence $S_{m} \neq S_{m-1} S=S_{m-1}$ is a subgroup of $G$. We may thus assume that $S_{m-1}=S_{m}=S_{m-1} S$, which means that $S_{m}$ is closed under multiplication and hence it is a subgroup of $G$.

Both hypotheses in Theorem 2.1 are necessary. If $1 \notin S$, then the result is false for $m=1$; while if $S=\{1, a\}$, where $a$ is an element of order $m+2$, then $\left|S_{m}\right|=m+1$ and $S_{m}$ is not a group. It is convenient to state the following corollary.

2.2. Corollary. If $S$ is a set of generators of a group $G$ of order $m$ and $S$ contains the identity, then $\left|S_{l}\right|>l$ for all $l<m$; and in particular, $S_{m-1}=G$.

Proof. If we had $\left|S_{l}\right| \leqslant l$ for some $l<m$ then, by Theorem 2.1, $S_{l}$ would be the group generated by $S$, contrary to hypothesis. 
3. Applications to values of characters. Let $\chi$ be a character of order $k$.

3.1. Definition. Let $g_{m}=g_{m}(\chi)$ denote the least positive integer for which $\chi$ attains its $(m+1)$ st nonzero value. Thus $g_{0}(\chi)=1$ and $g_{m}(\chi)$ is the least positive integer with $\chi\left(g_{m}\right) \notin\left\{0, \chi\left(g_{0}\right), \ldots, \chi\left(g_{m-1}\right)\right\}$.

Now Theorem 2.1 leads to the following:

3.2. Lemma. If $x$ is a positive integer, $(x, n)=1$ and all prime divisors of $x$ lie in the interval $\left(1, g_{m}^{1 / m}\right)$, then $\chi(x)$ belongs to a subgroup of order $l \leqslant m, l \mid k$, of the group of kth roots of unity.

Proof. If we let $S=\left\{\chi(x) \mid(n, x)=1,0<x<g_{m}^{1 / m}\right\}$, then $S$ satisfies the hypotheses of Theorem 2.1 and $S_{m}$ is a subgroup contained in the set

$$
T=\left\{\chi(x) \mid(n, x)=1,0<x<g_{m}\right\} .
$$

3.3. LEMMA. Let $l$ be the order of the subgroup defined in the proof of Lemma 3.2.

(i) We have $l=1$ if and only if $g_{m} \leqslant g_{1}^{m}$.

(ii) If $1<l \leqslant m$, define $\chi_{1}=\chi^{l}$ as a character of order $k_{1}=k / l$. If $l=m$, then $g_{m}(\chi)=g_{1}\left(\chi_{1}\right)$.

(iii) If $1<l<m$, define

$$
m_{t}=[(m-l) /(t+1)]+1, \quad t=0,1, \ldots, l-1,
$$

and obtain

$$
g_{m}(\chi) \leqslant \min _{\substack{0<t<l \\ m_{1}<k_{1}}} g_{m_{1}}\left(\chi_{1}\right)^{m /(m-t)} .
$$

Proof. Part (i) is obvious, since $\chi(x)=1$ for $(x, n)=1,0<x<g_{m}^{1 / m}$, implies $g_{m}^{1 / m} \leqslant g_{1}$. Conversely, if $g_{m}^{1 / m} \leqslant g_{1}$, then $S=\{1\}=S_{m}$ so that $l=1$.

Part (ii) assumes that $l=m$. Thus $\chi(x)^{l}=1$ for every $0<x<g_{m},(x, n)$ $=1$ and $\chi\left(g_{m}\right)^{l} \neq 1$. Hence $g_{m}$ is the smallest positive integer $x,(x, n)=1$, for which $\chi_{1}(x) \neq 1$.

To prove part (iii), we wish to estimate the number of values attained by $\chi_{1}$ in the interval $\left[1, g_{m}(\chi)^{(m-t) / m}\right)$. Assume that $\chi_{1}(x)=\zeta$ for some $x$ with $(x, n)=1,1 \leqslant x<g_{m}(\chi)^{(m-t) / m}$. Since the set of values $\chi(y)$ with $1 \leqslant y<$ $g_{m}(\chi)^{1 / m},(n, y)=1$ generates a group of order $l$, we have

$$
\left|\left\{x\left(y_{1} \cdots y_{t}\right) \mid 1 \leqslant y_{1}, \ldots, y_{t}<g_{m}(\chi)^{1 / m},\left(y_{1} \cdots y_{t}, n\right)=1\right\}\right| \geqslant t+1
$$

for all $t<l$ by Corollary 2.2. Thus $\chi\left(x y_{1} \cdots y_{t}\right)$ attains at least $t+1$ distinct values $\zeta_{1}, \ldots, \zeta_{t+1}$ with $\zeta_{1}^{l}=\cdots=\zeta_{t+1}^{l}=\zeta$, for $1 \leqslant y_{1}, \ldots, y_{t}<$ $g_{m}(\chi)^{1 / m}$ and hence for $1 \leqslant x y_{1} \ldots y_{t}<g_{m}(\chi)$. In other words, to every nonzero value $\zeta$ attained by $\chi_{1}$ in the interval $\left[1, g_{m}(\chi)^{(m-t) / m}\right)$ there correspond at least $t+1$ distinct values of $\chi$, attained in the interval $\left[1, g_{m}(\chi)\right.$ ), whose $l$ th power is $\zeta$. In addition we know from Theorem 2.1 that all $l$ values 
of the subgroup of $l$ th roots of unity, that is the values $\chi(x)$ for which $\chi_{1}(x)=1$, are attained in the interval $\left[1, g_{m}(\chi)\right)$. Hence the number of distinct nonzero values of $\chi_{1}$ in $\left[1, g_{m}(\chi)^{(m-t) / m}\right)$ is at most $1+(m-l) /(t+1)$, that is

$$
\left|\left\{\chi_{1}(x) \mid(x, n)=1,0<x<g_{m}(\chi)^{(m-t) / m}\right\}\right| \leqslant\left[\frac{m-l}{t+1}\right]+1=m_{t}
$$

for $0 \leqslant t \leqslant l-1$. Hence $g_{m_{1}}\left(\chi_{1}\right) \geqslant g_{m}(\chi)^{(m-t) / m}$ for all these values of $t$ whenever the sumbol $g_{m_{t}}\left(\chi_{1}\right)$ makes sense.

Lemma 3.3 enables us to get upper bounds for $g_{m}(\chi)$ in terms of $g_{1}\left(\chi^{l}\right)$ for the divisors $l$ of $k$. If $k$ is a prime, or generally when $m$ is smaller than the least nontrivial divisor of $k$, then

$$
g_{m}(\chi) \leqslant g_{1}(\chi)^{m} .
$$

Now take $k=p^{2}$ where $p$ is an odd prime. Then $g_{m}(\chi) \leqslant g_{1}(\chi)^{m}$ for $m<p$. For $p \leqslant m \leqslant 2 p-1$, we have either $l=1$ and $g_{m} \leqslant g_{1}^{m}$ or $l=p$. In case $l=p$, choose $t=[m / 2]$. Then

$$
m_{t}=\left[\frac{m-p}{[m / 2]+1}\right]+1=1
$$

and

$$
g_{m}(\chi) \leqslant \max \left\{g_{1}(\chi)^{m}, g_{1}\left(\chi^{p}\right)^{m /(m-[m / 2])}\right\} \leqslant \max \left\{g_{1}(\chi)^{m}, g_{1}\left(\chi^{p}\right)^{2}\right\} .
$$

If $k>m \geqslant 2 p$ and $l=p$ choose $t=p-1$. Then $m_{t}=[m / p]$ and

$$
g_{m}(\chi) \leqslant \max \left\{g_{1}(\chi)^{m}, g_{[m / p]}\left(\chi^{p}\right)^{m /(m-p+1)}\right\} .
$$

But

$$
g_{[m / p]}\left(\chi^{p}\right) \leqslant g_{1}\left(\chi^{p}\right)^{[m / p]} \leqslant g_{1}\left(\chi^{p}\right)^{m / p}
$$

and therefore

$$
g_{m}(\chi) \leqslant \max \left\{g_{1}(\chi)^{m}, g_{1}\left(\chi^{p}\right)^{m^{2} / p(m-p+1)}\right\} .
$$

The computations get increasingly cumbersome as $k$ has more factors. However, since, as we observed in the introduction, every $\chi$ can be expressed as a product of characters of prime power order, it is particularly useful to give the relations for $k=p^{r}, p$ prime. This can be done by case divisions as above for the cases $r=1,2$. We omit the details.

Another upper bound for $g_{m}$ for $\chi$ of arbitrary order $k$ is obtained as follows.

Let $l_{1}$ be the order of the group generated by $\chi\left(g_{1}(\chi)\right)$. Define $\chi_{1}=\chi^{l_{1}}$ and let $l_{2}$ be the order of $\chi_{1}\left(g_{1}\left(\chi_{1}\right)\right)$ and so on. In this manner we get divisors $l_{1}$, $l_{2}, \ldots, l_{s}$ of $k$ so that $l_{i}>1$ and $l_{1} l_{2} \cdots l_{s}=k$; and characters $\chi_{i}=\chi^{l_{1} \cdots l_{i}}$ of order $k_{i}=k / l_{1} \cdots l_{i}=l_{i+1} \cdots l_{s}$. The order of the group $G_{i}$ generated by $\chi\left(g_{1}(\chi)\right), \chi\left(g_{1}\left(\chi_{1}\right)\right), \ldots, \chi\left(g_{1}\left(\chi_{i-1}\right)\right)$ is $l_{1} \cdots l_{i}$ as seen from the fact that 
$G_{i} / G_{i-1}$ is the cyclic group of order $l_{i}$ generated by $\chi\left(g_{1}\left(\chi_{i-1}\right)\right) G_{i-1}$, which is isomorphic to the group generated by $\chi_{i-1}\left(g_{1}\left(\chi_{i-1}\right)\right)$. All values of $G_{i}$ are attained by $\chi(x)$ with

$$
1 \leqslant x \leqslant g_{1}(\chi)^{l_{1}-1} g_{1}\left(\chi_{1}\right)^{l_{2}-1} \cdots g_{1}\left(\chi_{i-1}\right)^{l_{i}-1} .
$$

The values $\chi\left(g_{1}(\chi)^{a_{1}} g_{1}\left(\chi_{1}\right)^{a_{2}} \cdots g_{1}\left(\chi_{i-1}\right)^{a_{i}}\right)$ are distinct elements of $G_{i}$ for $0 \leqslant a_{j}<l_{j}$ and thus we have the following.

3.5. THEOREM. There exist divisors $l_{1}, l_{2}, \ldots, l_{s}$ of $k$ so that $l_{i}>1, l_{1} \cdots l_{s}$ $=k, \chi_{i}=\chi^{l_{1} \cdots l_{i}}$ and

$$
g_{m}(\chi) \leqslant g_{1}(\chi)^{a_{1}} g_{1}\left(\chi_{1}\right)^{a_{2}} \cdots g_{1}\left(\chi_{s-1}\right)^{a_{s}}, \quad 0 \leqslant a_{i}<l_{i},
$$

whenever

$$
\begin{aligned}
m \leqslant \mid\left\{\left(b_{1}, \ldots, b_{s}\right) \mid 0 \leqslant b_{i}<l_{i}\right. & g_{1}(\chi)^{b_{1}} \cdots g_{1}\left(\chi_{s-1}\right)^{b_{s}} \\
& \left.<g_{1}(\chi)^{a_{1}} \cdots g_{1}\left(\chi_{s-1}\right)^{a_{s}}\right\} \mid .
\end{aligned}
$$

Proof. We need only verify the assertion that

$$
\chi\left(g_{1}(\chi)^{a_{1}} \cdots g_{1}\left(\chi_{i-1}\right)^{a_{i}}\right)=\chi\left(g_{1}(\chi)^{b_{1}} \cdots g_{1}\left(\chi_{i-1}\right)^{b_{i}}\right)
$$

with $0 \leqslant a_{j}, b_{j}<l_{j}(j=1, \ldots, i)$ implies $a_{j}=b_{j}$ for $j=1, \ldots, i$. We prove this by induction on $i$. For $i=1$ we know that $\chi\left(g_{1}(\chi)\right)$ is a root of unity of order $l_{1}$, and hence $\chi\left(g_{1}(\chi)\right)^{a_{1}}=\chi\left(g_{1}(\chi)\right)^{b_{1}}$ implies $a_{1} \equiv b_{1}\left(\bmod l_{1}\right)$, therefore $a_{1}=b_{1}$ since $\left|a_{1}-b_{1}\right|<l_{1}$. Now assume the statement true for $i-1$ and raise both sides of (3.6) to the power $l_{1} \cdots l_{i-1}$ to get

$$
\chi_{i-1}\left(g_{1}\left(\chi_{i-1}\right)\right)^{a_{i}}=\chi_{i-1}\left(g_{1}\left(\chi_{i-1}\right)\right)^{b_{i}}
$$

which implies $a_{i} \equiv b_{i}\left(\bmod l_{i}\right)$ and hence $a_{i}=b_{i}$, since $\left|a_{i}-b_{i}\right|<l_{i}$. Thus we can cancel the factor $\chi\left(g_{1}\left(\chi_{i-1}\right)\right)^{a_{i}}=\chi\left(g_{1}\left(\chi_{i-1}\right)\right)^{b_{i}}$ on both sides of (3.6) and get $a_{j}=b_{j}(j=1, \ldots, i)$ by the induction hypothesis.

4. Bounds in terms of powers of the modulus. Using the results on character sums due to D. A. Burgess [1] and K. Norton [4] which show that the different values of $\chi$ are equally distributed in relative short intervals, together with a simple sieve argument first used by Vinogradov [5], one can get bounds for $g_{1}(\chi)$, and thus for general $g_{m}(\chi)$ whose order is a fractional power of $n$. For details and further references see the monograph of $\mathbf{K}$. Norton [3].

We need the following fact (compare [4, Theorem 7.24]).

4.1. Lemma. Let $N_{m}(h)$ be the number of integers $x$ in $[1, h]$ for which $\chi(x)=\chi\left(g_{m}\right)$ where $\chi$ is a character of order $k(\bmod n)$. Then

$$
N_{m}(h)=(\varphi(n) / k n) h+O\left(h^{1-1 / r_{n}}{ }^{(r+1) / 4 r^{2}+e}\right)
$$

where $r$ is an arbitrary positive integer, $\varepsilon>0$, and the implied constant in $O$ depends only on $r$ and $\varepsilon$. 
Proof. Since Norton's proof in [4] refers to the case of power residues, we adapt the proof in [4] as suggested by the referee.

Let $\zeta$ be a $k$ th root of unity; then

$$
\frac{1}{k} \sum_{l=1}^{k} \zeta^{l}= \begin{cases}1 & \text { if } \zeta=1 \\ 0 & \text { if } \zeta \neq 1 .\end{cases}
$$

Hence

$$
\frac{1}{k} \sum_{l=1}^{k} \chi^{l}(x) \bar{\chi}^{l}\left(g_{m}\right)= \begin{cases}1 & \text { if } \chi(x)=\chi\left(g_{m}\right) \\ 0 & \text { otherwise. }\end{cases}
$$

Summing over $1 \leqslant x \leqslant h$, we get

$$
\begin{aligned}
N_{m}(h) & =\frac{1}{k} \sum_{l=1}^{k} \bar{\chi}^{l}\left(g_{m}\right) \sum_{1<x<h} \chi^{l}(x) \\
& =\frac{1}{k}\left\{\sum_{1<x<h} \chi^{k}(x)+\sum_{l=1}^{k-1} \bar{\chi}^{l}\left(g_{m}\right) \sum_{1<x<h} \chi^{l}(x)\right\} .
\end{aligned}
$$

Since $\chi^{k}$ is the principal character, we get by the method of Norton $[4$, p. 165] that

$$
\sum_{1<x<h} \chi^{k}(x)=\frac{\varphi(n)}{n} h+O\left(n^{\varepsilon}\right) .
$$

We now use Theorem 2 of [1] which gives

$$
\begin{array}{r}
\frac{1}{k}\left|\sum_{l=1}^{k-1} \bar{\chi}^{l}\left(g_{m}\right) \sum_{1<x<h} \chi^{l}(x)\right| \leqslant \frac{1}{k} \sum_{l=1}^{k-1}\left|\sum_{1<x<h} \chi^{l}(x)\right| \\
=\frac{1}{k} \sum_{l=1}^{k-1} O\left(h^{1-1 / r_{n}(r+1) / 4 r^{2}+\varepsilon}\right)=O\left(h^{1-1 / r_{n}} n^{(r+1) / 4 r^{2}+\varepsilon}\right)
\end{array}
$$

for any positive integer $r$ (in case $n$ is cube free as we assume throughout). Here the implied constant depends only on $r$ and $\varepsilon$.

Combining (4.3) and (4.4) we have the desired result.

We also need the Dickman function.

4.5. Definition. The Dickman function $\rho(\alpha)$ is defined for all nonnegative $\alpha$ by

$$
\begin{aligned}
& \rho(\alpha)=1, \quad 0 \leqslant \alpha \leqslant 1, \\
& \rho(\alpha)=\rho(N)-\int_{N}^{\alpha} \frac{\rho(\tau-1)}{\tau} d \tau, \quad \begin{array}{l}
N<\alpha \leqslant N+1, \\
N=1,2, \cdots .
\end{array}
\end{aligned}
$$

The function $\rho(\alpha)$ is monotonically strictly decreasing for $1 \leqslant \alpha<\infty$ with $\lim _{\alpha \rightarrow \infty} \rho(\alpha)=0$. It is therefore possible to define $\rho^{-1}(\zeta)$ for every $\zeta \in(0,1]$ as a number in [1, $\infty)$ (see [3, p. 3] for details).

Now let $N_{n}(x, y)$ be the number of integers prime to $n$ in the interval $[1, x]$ 
which have no prime divisors $>y$. Then (see $[3,(6.8)])$ for $\alpha>0, n \geqslant 3$ we have

$$
N_{n}\left(x, x^{1 / \alpha}\right)=\frac{\varphi(n)}{n} \rho(\alpha) x+O\left(\frac{(\log \log n)^{2} x}{\log x}+n^{e}\left(x^{1 / \alpha}+x^{1-1 / \alpha}\right)\right)
$$

where the implied constant depends only on $\alpha$ and $\varepsilon$.

We now write

$$
g_{m}(\chi)=n^{1 / \gamma_{m}(\chi)}, \quad m=1, \ldots, k-1 .
$$

In the following we assume $k$ fixed. Then if $h=n^{1 / 4+\varepsilon}$, we can choose $r$ such that the error term in (4.2) is small compared to the principal term. Thus

$$
\gamma_{m}>4-\varepsilon
$$

for all characters of order $k$ provided $n$ is sufficiently large.

Combining (4.2) and (4.6) we can prove the following (see [3, (1.7)]).

4.8. LEMMA. For every $\varepsilon$ there exists an $n(\varepsilon)$ such that $\gamma_{1} \geqslant 4 \rho^{-1}(1 / k)-\varepsilon$ for all $n>n(\varepsilon, k)$.

Proof. Assume that there exists a $\delta>0$ such that $\gamma_{1}<4 \rho^{-1}(1 / k)-\delta$ for infinitely many $n$ and corresponding characters $\chi$. Then choose $h=n^{1 / 4+\varepsilon}$, where $\varepsilon$ is chosen so small that

$$
g_{1}=n^{1 / \gamma_{1}}>h^{1 /\left(\rho^{-1}(1 / k)-\varepsilon\right)}+1 \text {. }
$$

From (4.2) we get

$$
N_{0}(h)=(\varphi(n) / k n) h(1+o(1)) .
$$

From (4.6) we get

$$
\begin{aligned}
N_{n}\left(h, g_{1}-1\right) & >(\varphi(n) / n) \rho\left(\rho^{-1}(1 / k)-\varepsilon\right) h(1+o(1)) \\
& >(\varphi(n) / n)(1 / k+\varepsilon) h(1+o(1)),
\end{aligned}
$$

since $\rho$ is a decreasing function. But every positive integer $x$ which is prime to $n$ and has all its prime factors $<g_{1}$ obviously satisfies $\chi(x)=1$. Thus $N_{0}(h) \geqslant N_{h}\left(h, g_{1}-1\right)$, in contradiction to (4.9) and (4.10).

In order to apply Lemma 4.8 to the estimates of $g_{m}$ which we obtained in $\$ 3$, we observe the following.

\subsection{Lemma. For all integers $k \geqslant 2$ and $1 \leqslant m<k$ we have}

$$
(1 / m) \rho_{1}^{-}(1 / k) \leqslant \rho^{-1}(m / k) .
$$

Proof. Set $f(x)=x \rho^{-1}(x / k)$. Then $f(x)$ is differentiable for all $x$ in $0<x<k$. For any $x$ in this range, write $y=\rho^{-1}(x / k)$, so that $f(x)=$ $k y \rho(y)$. Now, by Definition 4.3,

$$
\frac{d f}{d y}=k\left(\rho(y)+y \rho^{\prime}(y)\right)=k(\rho(y)-\rho(y-1))<0
$$

for all $y \in(1, \infty)$. Thus $f$ is a decreasing function of $y$ and hence an 
increasing function of $x$, so that $f(1) \leqslant f(m)$.

If we combine Lemma 4.11 with Theorem 3.5 and write

$$
g_{1}\left(\chi_{i}\right)=n^{1 / \gamma_{1}\left(\chi_{i}\right)},
$$

we find that for $i=0,1, \ldots, s-1$ and any $\varepsilon$, we have

for all large $n$.

$$
\begin{aligned}
\gamma_{1}\left(\chi_{i}\right) & >4 \rho^{-1}\left(1 / k_{i}\right)-\varepsilon=4 \rho^{-1}\left(\left(l_{1} \cdots l_{i}\right) / k\right)-\varepsilon \\
& \geqslant\left(4 / l_{1} \cdots l_{i}\right) \rho^{-1}(1 / k)-\varepsilon
\end{aligned}
$$

4.13. THEOREM. Let $k$ be a fixed integer. For every $\varepsilon$, there exists an $n(\varepsilon, k)$ such that for every character $\chi$ of order $k$, we have

$$
\gamma_{m}(\chi)>(4 / m) \rho^{-1}(1 / k)-\varepsilon
$$

for all $n>n(\varepsilon, k)$ and every integer $1 \leqslant m<k$.

Proof. If $k=1$ there is nothing to prove. If $k>1$ define the divisors $l_{1}, \ldots, l_{s}$ and the characters $\chi_{1}, \ldots, \chi_{s-1}$ as in Theorem 3.5. We shall prove that for any $\varepsilon$ there is an $n(\varepsilon, k)$ so that for all $n>n(\varepsilon, k)$ the number of solutions of the system

$$
g_{1}^{a_{1}}(\chi) g_{1}^{a_{2}}\left(\chi_{1}\right) \cdots g_{1}^{a_{s}}\left(\chi_{s-1}\right) \leqslant \nu^{m}, \quad 0 \leqslant a_{i}<l_{i}, \quad i=1, \ldots, s,
$$

where $\nu=n^{1 /\left(4 \rho^{-1}(1 / k)\right)+\varepsilon}$ is at least $m+1$. Since $\chi$ attains distinct values at the integers on the left side of (4.14), this proves the theorem.

If we set $g_{1}\left(\chi_{i}\right)=\nu^{\beta_{i}}, g_{1}(\chi)=\nu^{\beta}$, then from Lemmas 4.8 and 4.11 it follows that $\beta \leqslant 1, \beta_{i} \leqslant l_{1} \cdots l_{i}$. Now the number of solutions of (4.14) is equal to the number of solutions of

$$
a_{1} \beta+a_{2} \beta_{1}+\cdots+a_{s} \beta_{s-1} \leqslant m, \quad 0 \leqslant a_{i}<l_{i}, \quad i=1, \ldots, s,
$$

and the number of such solutions can only decrease if the $\beta, \beta_{i}$ are increased. It thus suffices to prove that the inequalities

$$
\begin{aligned}
a_{1}+a_{2} l_{1}+a_{3} l_{1} l_{2}+\cdots+a_{s} l_{1} l_{2} \cdots l_{s-1} & \leqslant m, \\
& 0 \leqslant a_{i}<l_{i}, \quad i=1, \ldots, s,
\end{aligned}
$$

have $m+1$ solutions. This is obvious from the fact that the expressions on the left of (4.16) represent all integers from 0 to $k-1=l_{1} \cdots l_{s}-1 \geqslant m$ in a unique manner as the $a_{i}$ vary in the given ranges.

Note that Theorem 4.13 is useful only for $m$ which are small compared to $k$ since $\rho^{-1}(1 / k)$ grows less rapidly than $k$. In fact (see [3, (3.24)]) we have $\rho^{-1}(1 / k)<<\log \mathrm{k} / \log \log k$ for large $k$.

While we do not know how to improve the results in Theorem 4.13 without better information on the character sums which appear in the proof of Lemma 4.1, it is possible to get additional information for $g_{m}$ of other characters in case $g_{1}(\chi)$ is comparatively large for some character $\chi$.

4.17. Corollary. Given characters $\chi_{1}$ of order $k_{1}$ and $\chi_{2}$ of order $k_{2}$. Assume that $g_{m}\left(\chi_{2}\right)<g_{1}\left(\chi_{1}\right)$. Then there exists an $n(\varepsilon, k)$ such that 


$$
g_{l}\left(\chi_{2}\right) \leqslant n^{\left(l / 4 \rho^{-1}(1 / k)\right)+\varepsilon}, \quad 1 \leqslant l \leqslant m,
$$

where $k=\left[k_{1}, k_{2}\right]$ and $n \geqslant n(\varepsilon, k)$.

Proof. Write $k_{1}=p_{1}^{\alpha_{1}} \cdots p_{s}^{\alpha_{s}}, k_{2}=p_{1}^{\beta_{1}} \cdots p_{s}^{\beta_{s}}$ and set $k_{1}^{\prime}=\Pi_{\alpha_{i}>\beta_{i}} p_{i}^{\alpha_{i}}$, $k_{2}^{\prime}=\Pi_{\beta_{i}>\alpha_{i}} p_{i}^{\beta_{i}}$. Then the character

$$
\chi=\chi_{1}^{k_{1} / k_{1}^{\prime}} \chi_{2}^{k_{2} / k_{2}^{\prime}}
$$

has order $k=k_{1}^{\prime} k_{2}^{\prime}=\left[k_{1}, k_{2}\right]$.

Now assume that $g_{1}\left(\chi_{1}\right)>g_{l}\left(\chi_{2}\right)$. Then in the interval $\left[1, g_{l}\left(\chi_{2}\right)\right)$ the character $\chi$ can assume at most $l$ distinct nonzero values. Hence $g_{l}\left(\chi_{2}\right) \leqslant$ $g_{l}(\chi)$. The corollary now follows from Theorem 4.13. It constitutes an improvement unless $k_{1}$ divides $k_{2}$.

5. More elaborate sieve arguments. We can improve the argument that led to the estimate of $g_{1}$ by way of (4.6) in a manner that was used by J. H. Jordan [2]. We illustrate the idea by estimating $g_{2}$.

5.1. Definition. Let $N(x, y, z)$ be the number of integers in $[1, x]$ which have no prime factor $\geqslant y$ and at most one prime factor $>z$. Define $N_{n}(x, y, z)$ is an analogous manner, restricting attention to the integers which are relatively prime to $n$.

5.2. Definition. $\rho(\alpha, \beta)=\rho(\beta)+\int_{\alpha}^{\beta} \rho(\beta-\beta / \tau) d \tau / \tau$, where $1 \leqslant \alpha \leqslant \beta$ $<\infty$.

5.3. LEMma. $N\left(x, x^{1 / \alpha}, x^{1 / \beta}\right)=\rho(\alpha, \beta) x+o(x)$, where the constant implied in $o(x)$ depends only on $\alpha, \beta$.

Proof. We have

$$
\begin{aligned}
N\left(x, x^{1 / \alpha}, x^{1 / \beta}\right) & =N\left(x, x^{1 / \beta}\right)+\sum_{x^{1 / \beta}<p<x^{1 / \alpha}} N\left[\left(\frac{x}{p}\right), x^{1 / \beta}\right] \\
& =x \rho(\beta)+\sum_{x^{1 / \beta}<p<x^{1 / \alpha}} \frac{x}{p} \rho\left(\beta \frac{\log (x / p)}{\log x}\right)+o(x) \\
& =x\left(\rho(\beta)+\int_{x^{1 / \beta}}^{x^{1 / \alpha}} \rho\left(\beta \frac{\log (x / t)}{\log x}\right) \frac{d t}{t \log t}+o(1)\right) \\
& =x\left(\rho(\beta)+\int_{\alpha}^{\beta} \rho\left(\beta-\frac{\beta}{\tau}\right) \frac{d \tau}{\tau}\right)+o(x) \\
& =x \rho(\alpha, \beta)+o(x)
\end{aligned}
$$

where we have substituted $t=x^{1 / \tau}$ in the first integral.

We shall actually use a more specific lemma which we state without proof since the argument is entirely analogous to the one in [3] which leads to (4.6).

5.4. LEMMA. If $x>n^{\delta}$ for $a$ fixed $\delta>0$ and $\beta>\alpha \geqslant 1$, then 


$$
N_{n}\left(x, x^{1 / \alpha}, x^{1 / \beta}\right)=(\varphi(n) / n) \rho(\alpha, \beta) x(1+o(1)) .
$$

Now we can express a bound for $g_{2}$.

5.5. THEOREM. Let $\chi$ be a character of order $k(\bmod n)$, and let $\gamma_{1}=\gamma_{1}(\chi)$, $\gamma_{2}=\gamma_{2}(\chi)$ be as in $\$ 4$. Then for any $\varepsilon$ and all large $n$, we have

$$
\rho\left(\gamma_{2} / 4, \gamma_{1} / 4\right)<2 / k+\varepsilon .
$$

Since $\gamma_{1} / 4>\rho^{-1}(1 / k)-\varepsilon$ for all large $n$ and $\rho(\alpha, \beta)$ is a decreasing function of $\beta$ for $1 \leqslant \alpha \leqslant \beta<\infty$, we get

$$
\rho\left(\gamma_{2} / 4, \rho^{-1}(1 / k)\right)<2 / k+\varepsilon
$$

for all large $n$.

Proof. Choose $h=n^{1 / 4+\varepsilon}$. According to Lemma 4.1, the number of integers in $[1, h]$ for which $\chi$ has the value 1 or $\chi\left(g_{1}\right)$ is $(2 \varphi(n) / k n) h(1+$ $o(1))$.

Now every integer $x$ in $[1, h]$ with $(x, n)=1$, which has no prime factor $\geqslant g_{2}$ and has at most one prime factor $\geqslant g_{1}$, obviously has $\chi(x)=1$ or $\chi(x)=\chi\left(g_{1}\right)$. The number of such integers is

$$
N\left(h, n^{1 / \gamma_{2}}, n^{1 / \gamma_{1}}\right)>N\left(h, h^{4 / \gamma_{2}+\varepsilon}, h^{4 / \gamma_{1}+\varepsilon}\right)>\frac{\varphi(n)}{n}\left(\rho\left(\frac{\gamma_{2}}{4}, \frac{\gamma_{1}}{4}\right)-\varepsilon\right) h .
$$

Thus

$$
\frac{2 \varphi(n)}{k n} h>\frac{\varphi(n)}{n}\left(\rho\left(\frac{\gamma_{2}}{4}, \frac{\gamma_{1}}{4}\right)-\varepsilon\right) h
$$

and the result follows.

It would not be difficult to extend these arguments to estimate $g_{m}$ for larger values of $m$, but it would involve more case divisions.

\section{REFERENCES}

1. D. A. Burgess, On character sums and L-series. II, Proc. London Math. Soc. (3) 13 (1963), $524-536$.

2. J. H. Jordan, The distribution of cubic and quintic non-residues, Pacific J. Math. 16 (1966), 77-85.

3. K. K. Norton, Numbers with small prime factors and the least kth power non-residue, Mem. Amer. Math. Soc. No. 106 (1971).

4. Upper bounds for $k$-th power coset representatives modulo n, Acta. Arith. 15 (1969), 161-179.

5. I. M. Vinogradov, On the bound of the least non-residue of nth powers, Bull. Acad. Sci. USSR 20 (1926), 47-58 (Trans. Amer. Math. Soc. 29 (1927), 218-226).

Department of Mathematics, University of California, los Angeles, California 90024 (Current address of E. G. Straus)

Current address (G. Kolesnik): Department of Mathematics, The University of Texas at Austin, RLM 8-100, Austin, Texas 78712 